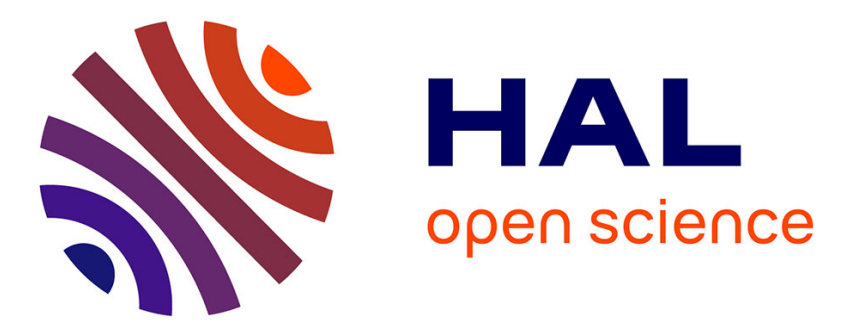

\title{
Ab-initio calculations of hydrogen diffusion coefficient in monoclinic zirconia
}

Orane Barbour, Jean-Paul Crocombette, Thomas Schuler, Marc Tupin

\section{To cite this version:}

Orane Barbour, Jean-Paul Crocombette, Thomas Schuler, Marc Tupin. Ab-initio calculations of hydrogen diffusion coefficient in monoclinic zirconia. Journal of Nuclear Materials, 2020, 539, pp.152333. 10.1016/j.jnucmat.2020.152333 . cea-03169490

\section{HAL Id: cea-03169490 https://hal-cea.archives-ouvertes.fr/cea-03169490}

Submitted on 15 Mar 2021

HAL is a multi-disciplinary open access archive for the deposit and dissemination of scientific research documents, whether they are published or not. The documents may come from teaching and research institutions in France or abroad, or from public or private research centers.
L'archive ouverte pluridisciplinaire HAL, est destinée au dépôt et à la diffusion de documents scientifiques de niveau recherche, publiés ou non, émanant des établissements d'enseignement et de recherche français ou étrangers, des laboratoires publics ou privés.

\section{(1) (1) $\$$}

Distributed under a Creative Commons Attribution - NonCommercial - NoDerivatives| 4.0 


\title{
Ab-initio calculations of hydrogen diffusion coefficient in monoclinic zirconia
}

\author{
Orane Barbour ${ }^{1}$, Jean Paul Crocombette ${ }^{1}$, Thomas Schuler ${ }^{1}$ and Marc Tupin ${ }^{2}$, \\ ${ }^{1}$ DEN-Service de Recherche en Métallurgie Physique, CEA, Université Paris-Saclay, F-91191 Gif-sur- \\ Yvette Cedex, France \\ ${ }^{2} D E N$-Service d'Etudes des Matériaux Irradiés, CEA, Université Paris-Saclay, F-91191, Gif-sur-Yvette, \\ France
}

Corresponding author : Jean Paul Crocombette Tel/Fax :

\begin{abstract}
During the corrosion in primary water of zirconium, hydrogen from the water diffuses through the oxide. To better understand this process, we use Density Functional Theory with hybrid functionals to calculate the energetics of interstitial hydrogen ions in defect-free monoclinic zirconia. While there is only one stable site for hydride ions in zirconia, protons have four different sites. We calculate the migration paths and energies between insertion sites to obtain the diffusion coefficients of hydrogen. We find that protons diffuse orders of magnitude faster than hydride ions, proving that protons are responsible for diffusion of hydrogen in monoclinic zirconia.
\end{abstract}




\section{I.Introduction}

Hydrogen is usually considered as an undesirable impurity in many materials such as nickel-base alloy, stainless steel, zirconium alloys because hydrogen tends to embrittle the materials even at low concentration by acting on the strength of the chemical binding as well as to act as carrier trap in semiconductor.

In Pressurized Water Reactors, zirconium alloys are used as fuel rod cladding materials. These claddings form the first containment barrier to fission products and their mechanical integrity has to be ensured during their lifetime in reactor. They are exposed in service to aggressive aqueous environment. In these conditions, the oxidation kinetics of Zircaloy-4 cladding alloy show a significant acceleration above $35 \mathrm{GWd} / \mathrm{tU}$ burnup [1]. As a consequence, the corrosion of Zircaloy-4 is one of the main limiting factors of the fuel rod lifetime. According to literature data, this enhanced corrosion rate would be due to the precipitation of hydrides at the metal/oxide interface in the metallic part of the cladding [2] [3] [4] [5, 6] [7] [8]. Indeed, during the corrosion of the cladding in primary water, a fraction of the hydrogen from the water involved in the oxidation reaction goes across the oxide layer up to the alloy. Once its solubility limit in the matrix is reached at the irradiation temperature in reactor core, hydrogen precipitates as brittle zirconium hydrides mainly located just under the metal/oxide interface. Another issue related to hydrogen transport through zirconium oxide is the potential release in dihydrogen gas form during the spent fuel transport. Hydrogen trapped within the oxide layer that grew in reactor core is indeed likely to be released from the spent fuel assembly into the transport conditioning. For this reason, there is a potential safety risk for the transport. Hydrogen desorption from the cladding is actually the opposite process of hydrogen absorption occurring during corrosion.

Irrespective of the process, absorption or desorption, hydrogen has to diffuse through the oxide layer towards the metal or the surface of the cladding. It was usually assumed that the diffusion process through the oxide layer was the rate-limiting step of hydrogen pick-up or of the desorption $[9,10]$. However, some researchers considered that hydrogen pick-up is controlled by the migration of hydrogen under an electric field built in the oxide layer [11].

Anyway, research was initially focused on hydrogen diffusion through the oxide layer. In the past, the diffusion coefficient of hydrogen in the corrosion film was widely investigated either by 1) isotopic tracers, or 2) hydrogen implantation followed by Secondary lon Mass Spectrometry (SIMS) or nuclear reaction analysis (NRA) [12] [13] [14] [15] [9] [16] [17] [18] [19] . However, due to the presence of two sub-layers in the oxide with different hydrogen transport properties [12], as well as the two types of hydrogen interaction within the oxide [9] or the polycrystalline structure of the oxide layer [19], apparent diffusion coefficient of hydrogen is difficult to evaluate accurately from measurements. The diffusion coefficients measured in the literature are very scattered for the Zircaloy- 4 alloy and to a lesser extent for $\mathrm{Zr}-\mathrm{Nb}$ alloy. They rank from $10^{-16}$ to $10^{-13} \mathrm{~cm}^{2} \mathrm{~s}^{-1}$ at $285^{\circ} \mathrm{C}$.

Sundell et al. [20] showed by Atom Probe Tomography that hydrogen in the oxide layer is mainly located and concentrated in the grain boundaries compared to the volume of the grains. Hydrogen transport in the oxide is thus a mixed diffusion process through the grain boundaries and the grains. Because of the very low diameter ( $35 \mathrm{~nm}$ ) of the columnar oxide grains[21], mesoscopic numerical calculations of grain boundary and bulk diffusion coefficients from fitting hydrogen diffusion profiles did not lead to accurate values of these coefficients. Finally, the charge state of hydrogen in monoclinic zirconia during corrosion in primary water is not clearly identified. Although recent study suggested that hydrogen could diffuse in the molecular form through nano-pipes in the oxide layer [22], it is usually considered in the corrosion modelling that hydrogen is in the proton form in the oxide layer as it comes from water molecules involved in the corrosion process [23] [24] [8].

For all these reasons, it is relevant to perform Density Functional Theory (DFT) calculations in order to know the charge state of hydrogen in zirconia and the hydrogen diffusion coefficient in the volume of 
the monoclinic zirconia grains. The present study is restricted to hydrogen in interstitial positions in pure monoclinic zirconia $\left(\mathrm{m}-\mathrm{ZrO}_{2}\right)$. Hydrogen is indeed usually found to be an interstitial impurity in many materials $[25,26]$. Zirconia being an insulator, hydrogen interstitials are expected to introduce defect levels in the insulating gap. It is therefore important to describe correctly this gap. In order to do so, our calculations were performed with the HSE06 [27] Hybrid Functional (HF).

A few studies are available in literature on this subject. They deal with various phases of zirconia (monoclinic, tetragonal, stabilized with an aliovalent cation). Most use Generalized Gradient Approximation (GGA) functionals and thus describe poorly the gap of zirconia and the hydrogen defect levels in this gap[28-35]. Some studies are dedicated to hydrogen diffusion in the zirconia lattice. Some authors have indeed performed calculations of hydrogen diffusion coefficients in tetragonal or monoclinic zirconia using GGA functionals [33] [30]. A couple of hybrid functional calculations [36, 37] are available but only one[37] considers monoclinic zirconia and it does not deal with the diffusion of hydrogen.

We will discuss our results in view of the previous works in the discussion section (see below).

In the present work, we carry out a systematic search of the insertion sites of hydrogen with various charge states in monoclinic zirconia within hybrid functional DFT in order to describe correctly the gap. We then calculate the migration paths between these sites and integrate the results to obtain the diffusion coefficients of hydrogen in defect-free monoclinic zirconia. The following section presents the technicalities of our calculations, results are then presented and we discuss them in the final part.

\section{II.Technicalities}

\section{Computational details}

We calculated hydrogen energetics in zirconia within the Density Functional Theory (DFT) theoretical framework. We used the VASP code (Vienna Ab initio Simulation Package) [38, 39]. In this code, electronic wave functions are expressed in a Projector Augmented Wave (PAW) [40] basis with 4s and $4 p$ semi core states included in the $\mathrm{Zr}$ valence configuration. Zirconia being an insulator, hydrogen interstitials are expected to introduce defect levels in the insulating gap. In order to evaluate accurately the gap, our calculations were performed with the HSE06 [27] Hybrid Functional (HF). We used GGA of the PBEO [41] type to perform initial relaxations then proceeded with further HF relaxation starting from GGA relaxed configurations. In some cases, as explained below, we also used GGA calculations to make a first selection of calculations before refining them with hybrid functionals.

Unless stated differently, electronic self consistency is achieved down to $10^{-4} \mathrm{eV}$ and atomic relaxations are converged down to an energy difference between two successive steps of $10^{-3} \mathrm{eV}$.

We first validated our approach on bulk properties of monoclinic zirconia [42], the unit cell of which is made of 12 atoms (4 formula units) After convergence tests, we chose a $600 \mathrm{eV}$ energy cut-off which warrants an energy convergence to less than $10^{-2} \mathrm{eV}$ for this unit cell. A $3 * 3 * 3$ regular $\mathrm{K}$ mesh was used for unit cell calculations. The obtained structural parameters are reproduced within $1 \%$ in GGA and within less than $0.2 \%$ for $\mathrm{HF}$ calculations As expected, GGA calculations underestimates the gap, predicted to be $3.4 \mathrm{eV}$ while the experimental values rank from 5.1 to $5.8 \mathrm{eV}$ [43]. HSE06 calculations correct the prediction with a $5.4 \mathrm{eV}$ gap. The present results prove to be in very good agreement with previous comparable calculations (e;g; Jaffe et al[44] for GGA and Lyons and van de Walle[37] for HF). 


\section{Hydrogen insertion procedure}

Hydrogen was then inserted in zirconia supercells. We performed most of the calculations on $2 \times 2 \times 2$ (96 atoms) supercells. We also performed calculations on $3 \times 3 \times 3$ (324 atoms) to check the convergence with respect to the supercell size. We found negligible differences between the results with the smaller supercell (see below). For supercell calculations, we restricted the K-point sampling to the $\Gamma$ point.

We performed a systematic search of hydrogen position in the zirconia crystalline lattice. We considered a mesh of regularly spaced possible insertion sites. The spacing of sites was chosen equal to $0.2 \AA$. Some distance restrictions were implemented to discard unphysical situations: Insertion sites closer than $0.9 \AA$ (resp. $1.5 \AA$ ) from oxygen (resp. zirconium) atoms were not considered. These thresholds were chosen considering the $\mathrm{H}-\mathrm{O}$ distance in the water molecule [45] (0.96 $\AA$ ) and the $\mathrm{Zr}-\mathrm{H}$ distance in some $\mathrm{Zr}-\mathrm{H}_{\mathrm{x}}$ complexes [46] (2.1 $\AA$ ). Furthermore, the symmetries of monoclinic zirconia enable to lower the number of calculations. Indeed each position within the unit cell is quadruplicated by symmetry. We could thus restrict the grid to $1 / 4$ of the unit cell, the rest of it being covered by symmetries. One obtains eventually 135 inequivalent possible insertion sites for hydrogen in monoclinic zirconia. One hydrogen atom is inserted in each of these sites and the atomic structure is relaxed as indicated above. For all sites, the three possible charge states of hydrogen are considered: $\mathrm{H}^{-}$(hydride ion), $\mathrm{H}^{0}$ (atomic hydrogen), and $\mathrm{H}^{+}$(proton) by changing the number of electrons in the box accordingly.

From the energies of the relaxed boxes, one can deduce the stability of the various charge states writing the energy of formation $\left(E^{f}\right)$ of an interstitial hydrogen as a function of its charge $(q=-1,0,+1)$ and the position of the Fermi level within the gap :

$$
E^{f}=E\left(H^{q}-\mathrm{ZrO}_{2}\right)-E\left(\mathrm{ZrO}_{2}\right)-\frac{1}{2} \mathrm{E}\left(\mathrm{H}_{2}\right)+q\left(\epsilon_{F}+\epsilon_{B V}\right)+\frac{q^{2} \alpha_{M}}{2 \varepsilon L}
$$

In this equation, $\left(\mathrm{H}-\mathrm{ZrO}_{2}\right), E\left(\mathrm{ZrO}_{2}\right)$ and $\mathrm{E}\left(\mathrm{H}_{2}\right)$ are the VASP energies of boxes containing hydrogen in zirconia, pure zirconia and an empty box with only an hydrogen molecule, respectively. The latter box provides the outer hydrogen reference. $\epsilon_{B V}$ is the energy of the top of the valence band and $\epsilon_{F}$ is a parameter indicating the energy of the Fermi level measured from the top of the valence band. The last term is the major correction of the energies of charged boxes [47]. It depends on the size of the box (L), the Madelung constant of the structure (calculated by VASP) and the dielectric constant of the material for which we take the 23 experimental value [48].

Finite temperature effects have been considered through the calculation of vibrational free energies in the harmonic approximation [49]. We made the further assumption that, because of hydrogen's very light weight, vibration modes of zirconium and oxygen atoms are not affected by the presence of hydrogen. We thus calculated the $3 \times 3$ force matrix from small displacements of hydrogen along the three axes. The diagonalization of this matrix gives the vibration frequencies of hydrogen. Stable positions have 3 frequencies $\omega_{i}$ which enter the vibrational free energies through:

$$
F^{\mathrm{vib}}=k T \sum_{i=1}^{3} \ln \left[2 \sinh \left(\frac{\hbar \omega_{i}}{2 k T}\right)\right]
$$

Where $\mathrm{k}$ is the Boltzmann constant, $\mathrm{T}$ is the temperature and $\mathrm{h}$ is the Planck constant. This energy should be added to $E^{\text {stable }}\left(\mathrm{H}^{q}-\mathrm{ZrO}_{2}\right)$ energy to compare the free energies of various sites for a given charge state, as a function of temperature. 


\section{Saddle point searches}

The migration energies and the associated migration paths between the various insertion sites of $\mathrm{H}$ in $\mathrm{ZrO}_{2}$ were calculated using the Nudged Elastic Band (NEB) method as implemented in VASP. We sampled the migration path with seven intermediate images. Unless otherwise stated, the spring constant was set to $5 \mathrm{eV} / \AA^{2}$. We first performed a standard NEB calculation [50]. Then we searched the actual position of the saddle point with a subsequent climbing NEB [51] calculation. For these calculations, the convergence criteria were decreased to $10^{-6} \mathrm{eV}$ for electronic self-consistency and $10^{-}$ ${ }^{5} \mathrm{eV}$ for atomic relaxation. The stable positions at both ends of the migration paths were also further relaxed with these more strict criteria. Vibrations at the saddle point were calculated in the same way as for stable positions. Saddle points have two positive vibration frequencies only. The jump frequency $(\Gamma)$ from a stable point through a saddle point depends[52] on the migration energy $\left(E_{\text {mig. }}\right)$ and the so-called attempt frequency $\left(\Gamma_{0}\right)$, through:

$$
\begin{gathered}
E_{\text {mig. }}=E^{\text {saddle }}\left(\mathrm{H}^{q}-\mathrm{ZrO}_{2}\right)-E^{\text {stable }}\left(\mathrm{H}^{q}-\mathrm{ZrO}_{2}\right) \\
\Gamma_{0}=\frac{\omega_{1, \text { stable }} \times \omega_{2, \text { stable }} \times \omega_{3, \text { stable }}}{\omega_{1, \text { saddlee }} \times \omega_{2, \text { saddle }}} \\
\Gamma=\Gamma_{0} \exp \left(-\frac{E_{\text {mig }}}{k T}\right) .
\end{gathered}
$$

We transferred the calculated jump frequencies and migration paths to the KineCluE code to obtain hydrogen diffusion coefficients. KineCluE[53,54] is an open-source code that implements the selfconsistent mean-field theory[55] in an automated and efficient way to compute transport coefficients from the atomic scale.

\section{III.Results}

\section{Hydrogen insertion sites}

Using GGA functional, the 135 insertion sites relax to a much smaller number of stable positions. One eventually obtains 8,10 and 7 stable sites for neutral, positive and negative hydrogen in zirconia, respectively. These positions are then recalculated with hybrid functional. The order of stability between the positions is mostly unaffected by the change of functional.

We first discuss the relative stability of the various charges of hydrogen in $\mathrm{ZrO}_{2}$. Using equation 1 for the insertion sites of lowest energy for each charge, one obtains that hydrogen is stable in positive or negative state for lower or higher Fermi level respectively (see figure 1). This so-called negative $U$ behaviour is very common for hydrogen in many materials [56]. The value of $U$ is defined as the energy difference between two neutral hydrogen and one pair of positive and negative hydrogen in the material.

$$
U=2 *\left(E(+/-)-E^{f}(0)\right)
$$

From table 1 , one extracts $\mathrm{U}=-2.44 \mathrm{eV}$ with hybrid functionals $(-1.36 \mathrm{eV}$ with $\mathrm{GGA})$. The energies of table 1 and figure 1 are obtained from equation 1 written with $1 / 2 \mathrm{E}\left(\mathrm{H}_{2}\right)$ as the reference hydrogen chemical potential. To get formation energies values closer to actual hydration energy, one can change this chemical potential to the one considering equilibrium with water and hydrogen molecules.

The hydrogen chemical potential is then

$$
\mu(\mathrm{H})=\frac{1}{2}\left(\mathrm{E}\left(\mathrm{H}_{2} \mathrm{O}\right)-\frac{1}{2} \mathrm{E}\left(\mathrm{O}_{2}\right)\right)
$$


Where $\mathrm{E}\left(\mathrm{H}_{2} \mathrm{O}\right)$ and $\mathrm{E}\left(\mathrm{O}_{2}\right)$ are VASP energies of water and dioxygen molecules respectively. With this reference the formation energies of figure 1 and table 1 should be increased by $+1.28 \mathrm{eV}$.

Neutral hydrogen energy is thus quite higher than energies of charged configurations at any Fermi level. The Charge Transition Levels (CTL) from positive to negative stable hydrogen for $2 * 2 * 2$ or $3 * 3 * 3$ super-cells and GGA or hybrid functionals are given in table 1, together with the formation energies of atomic hydrogen. For each functional the predicted energies in the smallest supercell are very close to one in the largest one, so that in the following calculations, we consider only the $2 * 2 * 2$ supercell. Furthermore, because atomic hydrogen are always less stable than positive and negative hydrogen, we restrict the subsequent calculations to positive and negative.

Figure 1: Formation energies (see Eq.1) of interstitial hydrogen as a function of Fermi level. Solid (resp. dotted) lines are for HSEO6 (resp GGA) calculations The $x$ scale goes from the top of the valence band to the bottom of the conduction band as calculated by HSEO6 functional. The dotted lines stop at the top of the GGA conduction band.
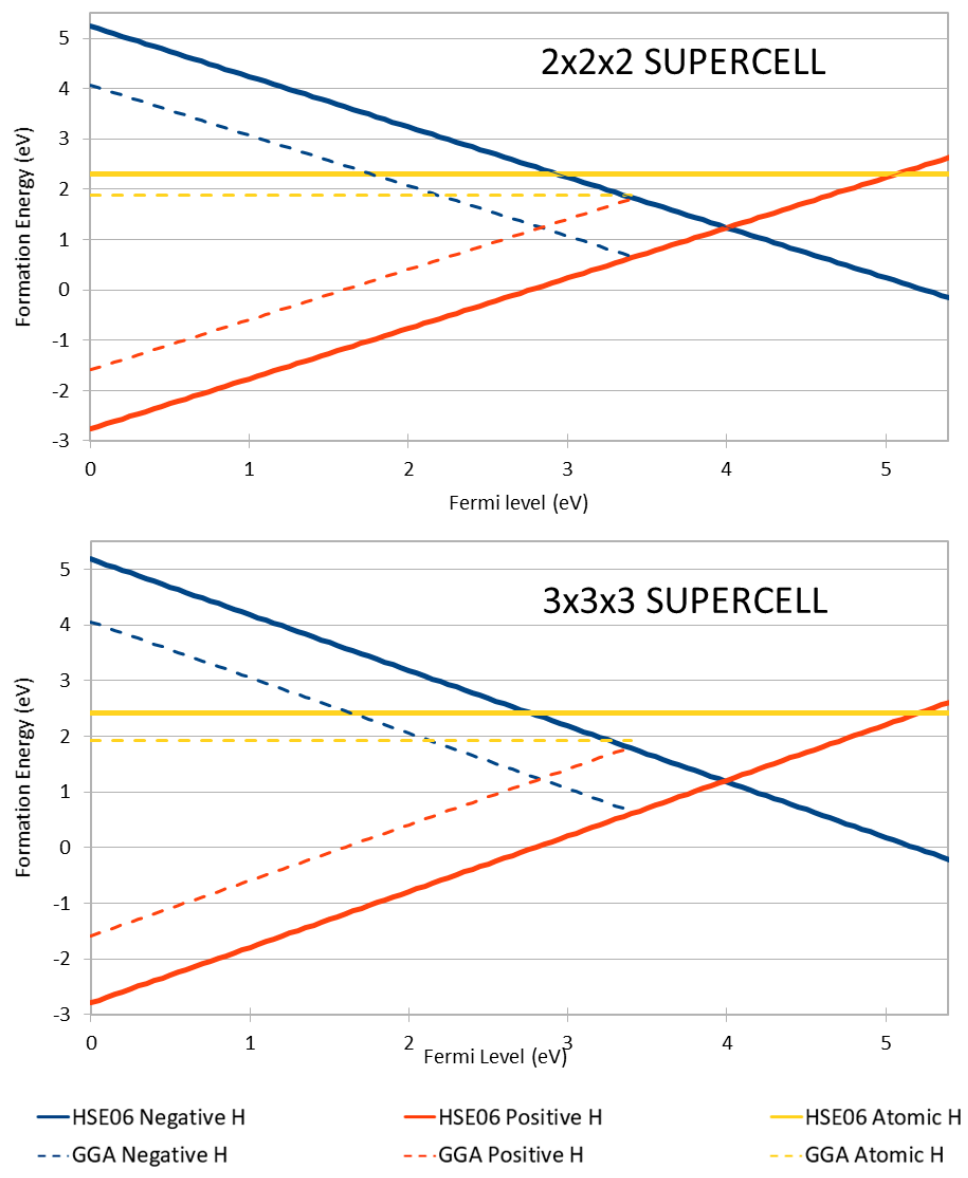
Table 1 : Characteristics of the formation energies of hydrogen in $\mathrm{ZrO}_{2}$ for various supercell sizes and exchange and correlation functionals (see text) extracted from Eq 1. The first and second lines describe the Charge Transition Level where negative and positive hydrogen ions have the same formation energy in zirconia. The last line indicates the formation energy of atomic hydrogen.

\begin{tabular}{lllll}
\hline & $\mathbf{2 \times 2 \times 2} \mathbf{~ G G A}$ & $\mathbf{3} \times \mathbf{3} \times \mathbf{3} \mathbf{G G A}$ & $\mathbf{2 \times 2 \times 2} \mathbf{F H}$ & $\mathbf{3 \times 3 \times 3} \mathbf{F H}$ \\
\hline +/- CTL Fermi level & $2,83 \mathrm{eV}$ & $2,82 \mathrm{eV}$ & $4,00 \mathrm{eV}$ & $3,99 \mathrm{eV}$ \\
\hline CTL Formation energy & $1,24 \mathrm{eV}$ & $1,24 \mathrm{eV}$ & $1,24 \mathrm{eV}$ & $1,20 \mathrm{eV}$ \\
\hline Formation energy of $\mathrm{H}^{0}$ & $1,89 \mathrm{eV}$ & $1,92 \mathrm{eV}$ & $2,31 \mathrm{eV}$ & $2,42 \mathrm{eV}$ \\
\hline
\end{tabular}

As mentioned above, hydride ions $\left(\mathrm{H}^{-}\right)$have seven final stable positions, see table 2a. They prove rather widespread in energy: the second lowest site is $0.5 \mathrm{eV}$ higher in energy than the minimum energy state, the subsequent sites being more than $2 \mathrm{eV}$ above in energy. All these subsequent states are neglected in the following migration paths calculations (see below). These path calculations evidenced that the second lowest site itself proves not to be a true local minimum but a saddle point with a quite flat energetic landscape where the atomic relaxation algorithm was stuck. It is thus discarder from the list of stable sites. To summarize on hydride ions, they have one stable insertion site in zirconia with an energy at least $2 \mathrm{eV}$ lower than any other metastable site. This site proves to be more or less equidistant from its closest oxygen and zirconium first neighbours (see table 2a). By application of the symmetries of the crystal this stable site is quadruplicated in the unit-cell of zirconia.

The situation is more complex for protons, see table $2 \mathrm{~b}$. In this case, five different inequivalent sites have energies within a $0.6 \mathrm{eV}$ range. The other sites lie higher in energy. One of the lowest energy sites proves to be a saddle point. Thus 4 inequivalent sites (labelled I to IV) remain, which amount to a total of 16 stable positions for protons in the unit cell of zirconia. For each site the 4 equivalent positions are labelled from 1 to 4 (e.g. $I_{1}$ to $I_{4}$ ). One can note that they tend to gather in thin slices perpendicular to the a direction, leaving a large portion of the structure devoid of stable proton positions, see figure 2.

We calculated the vibrational frequencies for the one negative site and its four positive counterparts see table 2. For $\mathrm{H}^{-}$the associated free energy is $+0.45 \mathrm{eV}$ at $285^{\circ} \mathrm{C}$. This energy shift is much lower that the energy difference with the site next in energy, which reinforces the neglect all the other high energy sites. For protons, the vibrational free energies are very close for the four sites, ranking from 0.29 to $0.31 \mathrm{eV}$. 
Table 2: Energy of stable sites of hydride ions (table 2a) and protons (table 2b) in monoclinic zirconia. For each charge, the zero is fixed at the energy of the most stable site. The sites with the highest energies, noted neg. have been neglected in free energy and migration paths calculations. Saddle $p t$. denotes positions that proved to be unstable saddle points in the migration paths calculations and neg. are positions high in energy which are neglected in the free energy and paths calculations.

2a:

\begin{tabular}{ccccc}
\hline \multicolumn{5}{c}{$\mathrm{H}^{-}$} \\
\hline label & energy $(\mathrm{eV})$ & Vib F $\left(285^{\circ} \mathrm{C}\right)$ & $1^{\text {st }} \mathrm{d}(\mathrm{H}-\mathrm{O})$ & $1^{\text {st }} \mathrm{d}(\mathrm{H}-\mathrm{Zr})$ \\
\hline saddle pt. & 0.45 & $0.22 \mathrm{eV}$ & $1.8 \AA$ & $2.1 \AA$ \\
\hline neg. & 2.30 & & & \\
\hline neg. & 2.47 & & \\
\hline neg. & 2.84 & & \\
\hline neg. & 2.92 & & \\
\hline neg. & 2.96 & & \\
\hline
\end{tabular}

2b:

\begin{tabular}{ccccc}
\hline \multicolumn{5}{c}{$\mathrm{H}^{+}$} \\
\hline label & energy $(\mathrm{eV})$ & $\mathrm{Vib} \mathrm{F}\left(285^{\circ} \mathrm{C}\right)$ & $1^{\text {st }} \mathrm{d}(\mathrm{H}-\mathrm{O})$ & $1^{\text {st }} \mathrm{d}(\mathrm{H}-\mathrm{Zr})$ \\
\hline $\mathrm{I}$ & 0 & 0.31 & 1.19 & 2.17 \\
\hline saddle $p t$. & 0.03 & 0.29 & 1.20 & 2.28 \\
\hline III & 0.23 & & & \\
\hline $\mathrm{IV}$ & 0.46 & 0.30 & 1.35 & 2.14 \\
\hline neg. & 0.68 & 0.30 & 1.24 & 2.09 \\
\hline neg. & 1.23 & & & \\
\hline neg. & 1.41 & & & \\
\hline neg. & 1.52 & & & \\
\hline neg. & 1.658972 & & & \\
\hline neg. & 2.597395 & & & \\
\hline
\end{tabular}

\section{Hydrogen migration paths}

The only stable site of $\mathrm{H}^{-}$in zirconia is multiplied by the translations and symmetry of the $\mathrm{ZrO}_{2}$ structure. In principle, one could consider all the atomic jumps between any pair of such sites. However, restricting the jump distances to a maximum of $4 \AA$ and considering symmetries one can reduce the number of inequivalent paths to four. Among these, one is found to have a very high saddle point in GGA and is neglected in the following. There are eventually three migration paths to calculate in hybrid functionals. These paths enable a 3-dimension migration of hydride ions in zirconia, see figure 4 . The corresponding migration energies are given in table 3a. Having calculated the two positive vibrational frequencies at each saddle point, we obtained the associated jump frequencies at $285^{\circ} \mathrm{C}$ given in table 3a. 
Table 3a : Migration between $\mathrm{H}^{-}$sites in zirconia.

\begin{tabular}{llll}
\hline & $\mathrm{I}_{1}-\mathrm{I}_{4}$ (short) & $\mathrm{I}_{1}-\mathrm{I}_{4}($ long $)$ & $\mathrm{I}_{1}-\mathrm{I}_{3}$ \\
\hline Migration energy & $0,45 \mathrm{eV}$ & $2,03 \mathrm{eV}$ & $2,26 \mathrm{eV}$ \\
\hline Jump frequency $\left(285^{\circ} \mathrm{C}\right)$ & $1,40.10^{10} \mathrm{~Hz}$ & $2,75.10^{-4} \mathrm{~Hz}$ & $2,50.10^{-7} \mathrm{~Hz}$ \\
\hline
\end{tabular}

Table 3b : Migration between $\mathrm{H}^{+}$sites in zirconia.

\begin{tabular}{|c|c|c|c|c|c|c|c|c|}
\hline & $I_{1}-I I_{1}$ & $I_{1}-I I_{4}$ & $I_{1}-I I_{3}$ & $I_{1}-I I I_{1}$ & $\mathrm{III}_{1}-\mathrm{II}_{4}$ & $\mathrm{IV}_{1}-\mathrm{IV}_{4}$ & $\mathrm{III}_{1}-\mathrm{IV}_{1}$ & $I_{1}-I V_{3}$ \\
\hline Migration & $I_{1}->I_{1}$ & $I_{1}->I_{4}$ & $I_{1}->I_{3}$ & $I_{1}->I I I_{1}$ & $\mathrm{III}_{1}<->\mathrm{III}_{4}$ & $\mathrm{IV}_{1}<->\mathrm{IV}_{4}$ & $\mathrm{III}_{1}->\mathrm{IV}_{1}$ & $\mathrm{II}_{1}->\mathrm{IV}_{3}$ \\
\hline \multirow[t]{3}{*}{ energy } & $0,34 \mathrm{eV}$ & $0,37 e V$ & $0,46 \mathrm{eV}$ & $0,61 \mathrm{eV}$ & 1,17eV & $0,69 \mathrm{eV}$ & $0,24 \mathrm{eV}$ & $0,74 \mathrm{eV}$ \\
\hline & $I_{1}->I_{1}$ & $I_{4}->I_{1}$ & $I_{3}->I_{1}$ & $\mathrm{III}_{1}->\mathrm{I}_{1}$ & & & $I V_{1}->I I I_{1}$ & $\mathrm{IV}_{3}->\mathrm{II}_{1}$ \\
\hline & $0,31 \mathrm{eV}$ & $0,34 \mathrm{eV}$ & $0,43 e V$ & $0,15 \mathrm{eV}$ & & & $0,03 \mathrm{eV}$ & $0,09 \mathrm{eV}$ \\
\hline \multirow{3}{*}{$\begin{array}{l}\text { Jump } \\
\text { frequency } \\
\left(285^{\circ} \mathrm{C}\right)\end{array}$} & $I_{1}->I_{1}$ & $I_{1}->I_{4}$ & $I_{1}->I I_{3}$ & $I_{1}->I I I_{1}$ & $\mathrm{III}_{1}<->\mathrm{III}_{4}$ & $\mathrm{IV}_{1}<->\mathrm{IV}_{4}$ & $\mathrm{III}_{1}->\mathrm{IV}_{1}$ & $\mathrm{II}_{1}->\mathrm{IV}_{3}$ \\
\hline & $1,24.10^{11} \mathrm{~Hz}$ & $6,99 \cdot 10^{10} \mathrm{~Hz}$ & $4,48 \cdot 10^{9} \mathrm{~Hz}$ & $7,37 \cdot 10^{8} \mathrm{~Hz}$ & $1,14.10^{4} \mathrm{~Hz}$ & $1,87 \cdot 10^{8} \mathrm{~Hz}$ & $7,97 \cdot 10^{11} \mathrm{~Hz}$ & $5,81 \cdot 10^{7} \mathrm{~Hz}$ \\
\hline & $\begin{array}{c}I_{1}->I_{1} \\
3,54 \cdot 10^{11} \mathrm{~Hz}\end{array}$ & $\begin{array}{c}I_{4}->I_{1} \\
1,99 \cdot 10^{11} \mathrm{~Hz}\end{array}$ & $\begin{array}{c}I_{3}->I_{1} \\
1,28 \cdot 10^{10} \mathrm{~Hz}\end{array}$ & $\begin{array}{c}I I I_{1}->I_{1} \\
9,10.10^{12} \mathrm{~Hz}\end{array}$ & & & $\begin{array}{c}I_{1}->I I_{1} \\
7,22 \cdot 10^{13} \mathrm{~Hz}\end{array}$ & $\begin{array}{c}\mathrm{IV}_{3}->\mathrm{II}_{1} \\
2.27 .10^{13} \mathrm{~Hz}\end{array}$ \\
\hline
\end{tabular}


Figure 2: Stable sites and migration paths for hydride ions in zirconia. Grey and red spheres represent zirconium and oxygen atoms respectively. $H^{-}$sites are in blue. Dotted lines indicate schematic migration paths. The color scheme for paths is consistent with the one of figure 4. For ease of reading, the $\mathrm{Zr}$ and $\mathrm{O}$ atomic positions are shown as they are in the perfect crystal. Actual jumps as determined by NEB calculations allow for change in the positions of the other atoms.
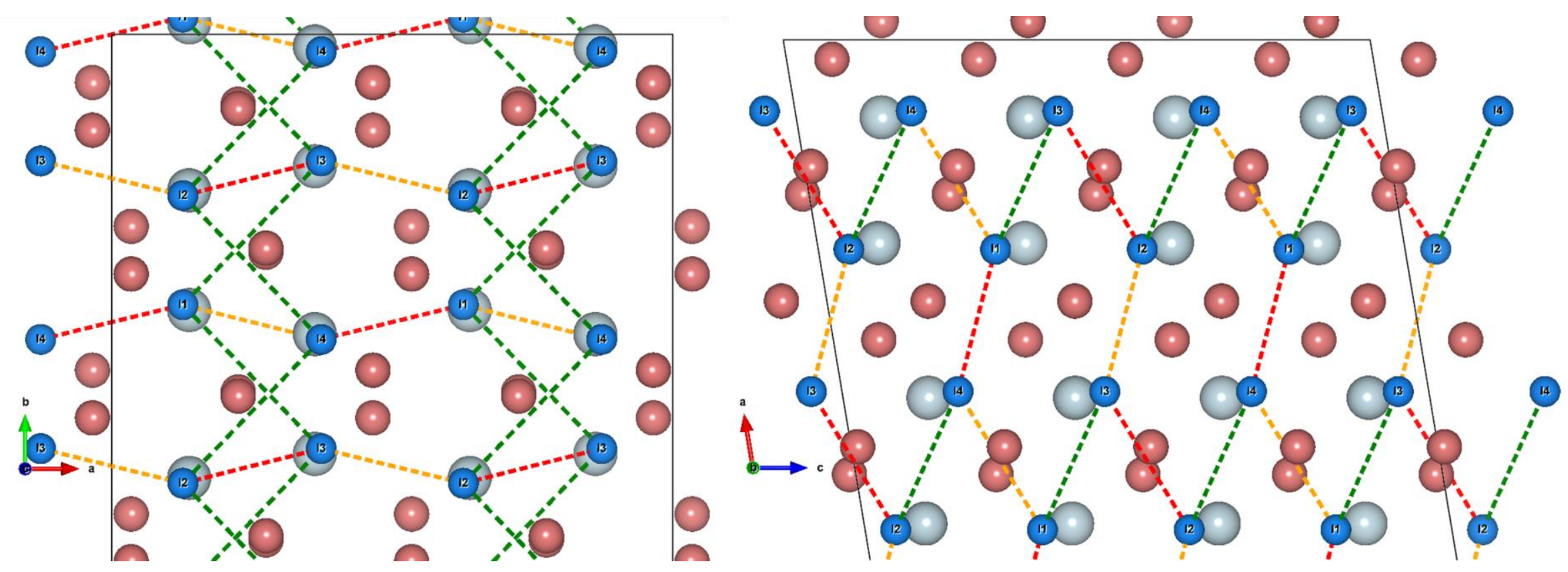
As discussed above protons have many more stable sites. To limit the number of calculations we neglected (like for hydride ions) paths with jump distances larger than $4 \AA$. As shown in figure 5 , stable sites accumulate in a thin slice perpendicular to the a direction. For jump within these slices, the paths with GGA migration barriers higher than $1 \mathrm{eV}$ were not calculated with hybrid functional and are neglected in the following. This criterion has not been considered for long distance inter slice jumps. Finally, some paths spontaneously decompose during NEB calculations into two smaller paths. They are not presented, as they are non-elemental migration pathways.

There are eventually eight different migration paths. To achieve a correct convergence of the NEB calculations we had to increase the spring constant to larger values ( 20 or $50 \mathrm{eV} / \AA^{2}$ depending on the path). Their energy profiles are presented in figure 5 . Some of these paths connect two inequivalent sites, the migration barrier is then non symmetric. In this case, the two jumps have two different migration energies and attempt frequencies depending on the direction of the jump (see table $3 \mathrm{~b}$ ). 
Figure 3: Stable sites and migration paths for protons in zirconia. Grey and red spheres represent zirconium and oxygen atoms respectively. $H^{+}$ sites of types I, II, III and IV are in green, blue, red and yellow, respectively. Dotted lines indicate schematic migration paths. The color scheme for paths is consistent with the one of figure 4. For ease of reading, the $\mathrm{Zr}$ and $\mathrm{O}$ atomic positions are shown as they are in the perfect crystal. Actual jumps as determined by NEB calculations allow for change in the positions of the other atoms.
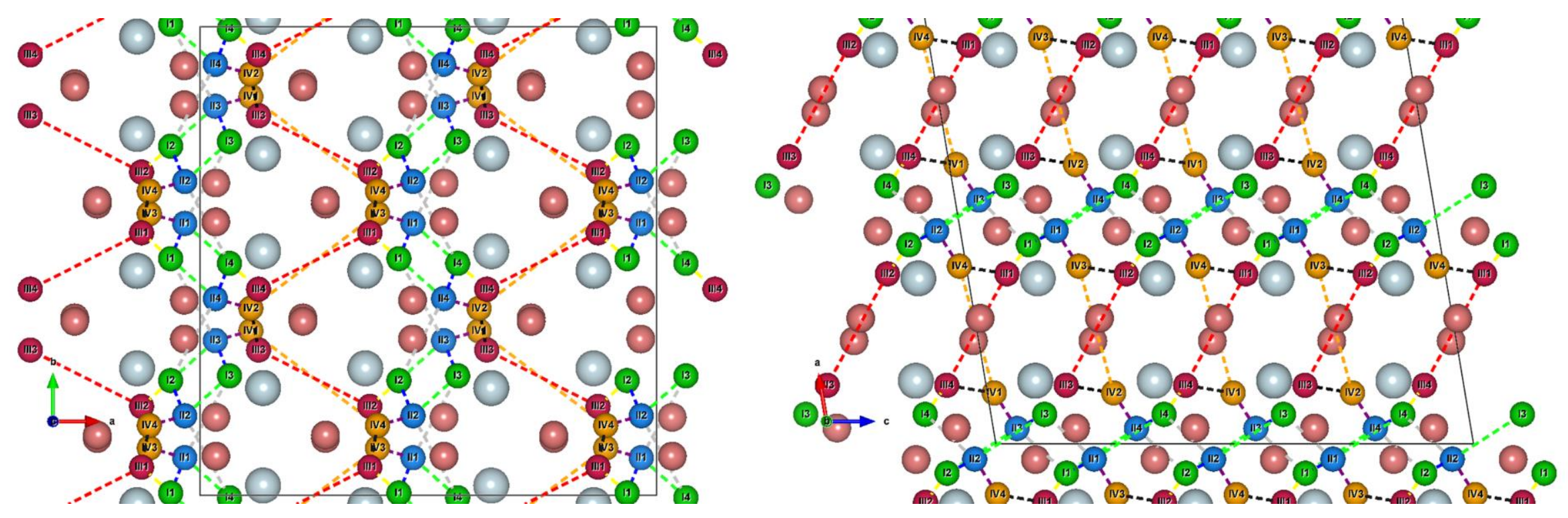
Figure 4: migration paths for $\mathrm{H}^{-}$hydride ions in $\mathrm{mZrO}_{2}$. The colour scheme is consistent with the one of dotted lines in figure 2.

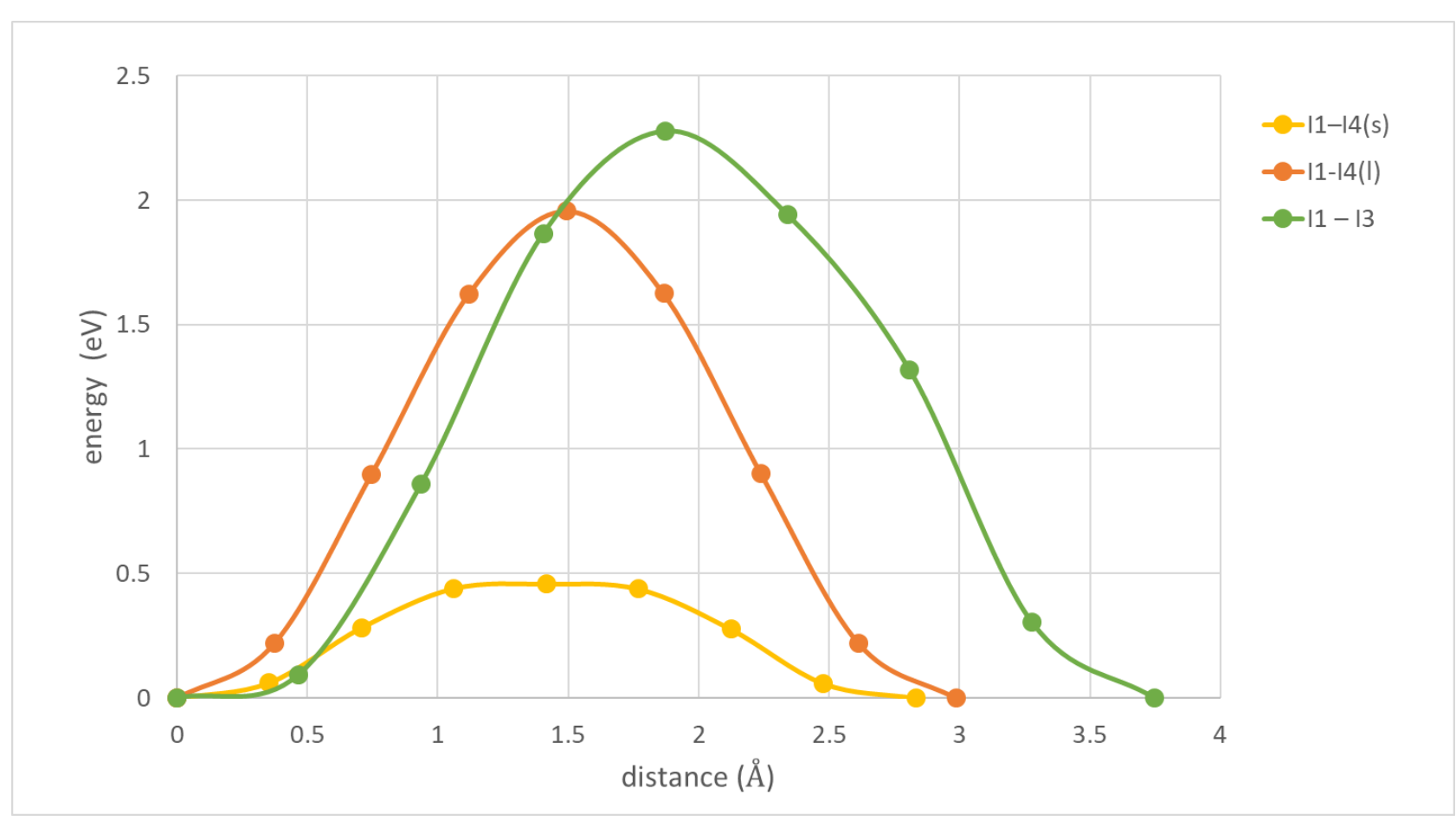


Figure 5: migration paths for $\mathrm{H}^{+}$protons in $\mathrm{mZrO}_{2}$. The colour scheme is consistent with the one of dotted lines in figure 3.

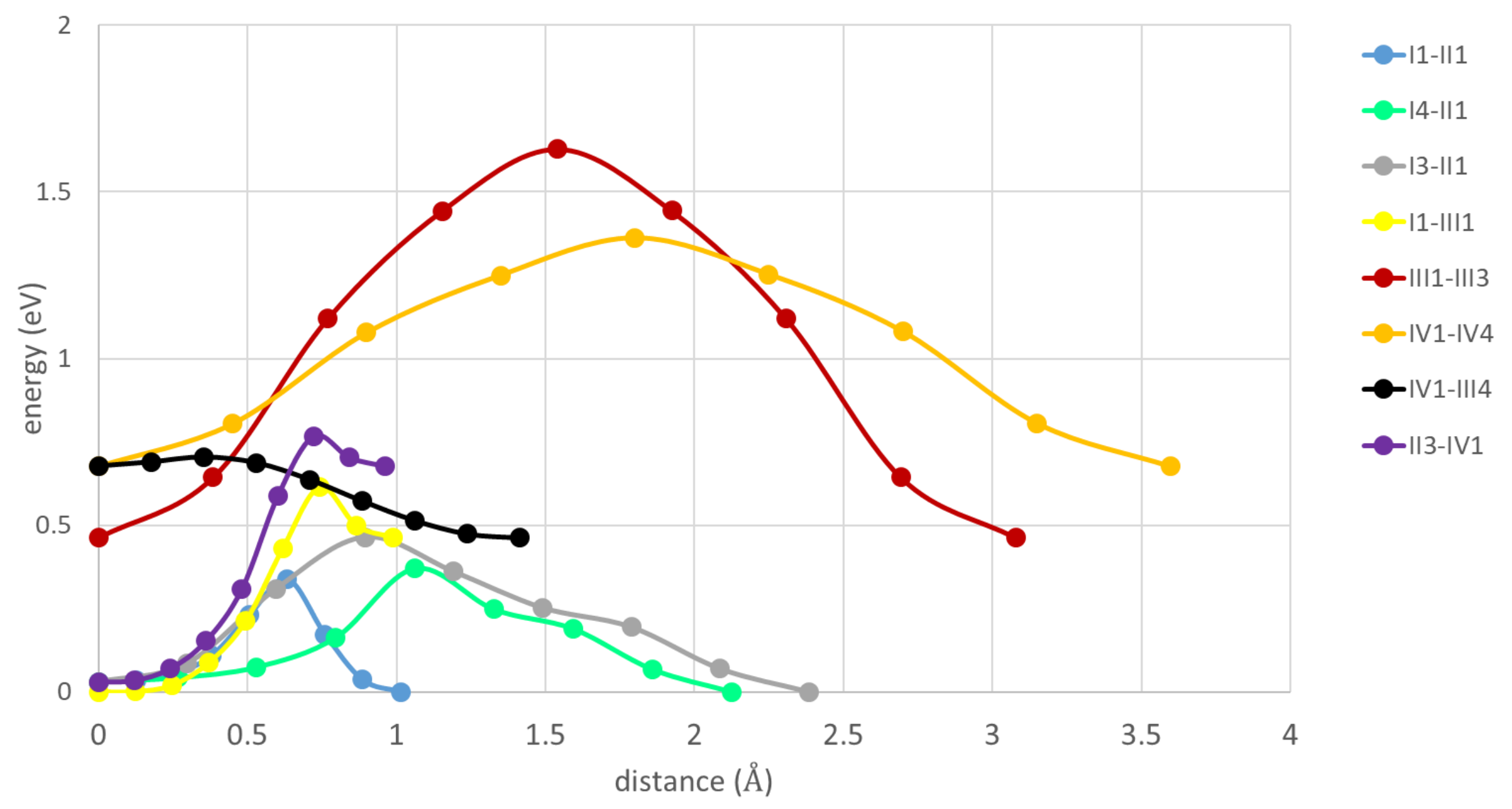




\section{Hydrogen diffusion coefficients}

The jump frequencies from site to site obtained with equations 3 to 5 are given in table 3 .

A quick look at this table shows that hydride ions diffusion should be much more difficult than that of protons.

To quantify this difference we used the KineCluE code to integrate all the information on jump frequencies and migration paths in various crystallographic directions. Beyond the three main directions, we also consider $(10 \overline{3})$ which is the main growth direction of the oxide grain on the cladding (ref. [20]). For hydride ions diffusion in monoclinic zirconia, we extracted the analytical expressions of the diffusion model from the code (see Appendix 1). For protons these expressions are too complicated to be useful due to the many available lattice sites and jump frequencies. The diffusion coefficients at $285^{\circ} \mathrm{C}$ along different directions are thus gathered in Table 4.

One can see that for protons diffusion is mainly $2 \mathrm{D}$ in the $(b, c)$ plane. At the opposite for hydride ions, diffusion is rather 1D with the fastest diffusion along the (a) direction. It appears clearly that hydride ions diffuse slower than protons. It is worth noting that for hydride ions, diffusing is much easier along the a direction while protons diffuses very quickly along the $b$ and $c$ directions compared to the $a$ direction.

Table 4 : Diffusion coefficients for hydride ions and protons in monoclinic zirconia at $285^{\circ} \mathrm{C}$.

\begin{tabular}{lcccc}
\hline Diffusion coefficient $\left(\mathrm{cm}^{2} . \mathrm{s}^{-1}\right)$ & \multicolumn{3}{c}{ Direction } \\
lon charge & $(001)$ & $(010)$ & $(100)$ & $(10 \overline{3})$ \\
$\mathrm{H}$ - (hydride) & $6.0010^{-21}$ & $6.5010^{-22}$ & $2.1110^{-19}$ & $4.1110^{-20}$ \\
$\mathrm{H}+$ (protons) & $2.6010^{-6}$ & $2.5610^{-6}$ & $7,0610^{-8}$ & $2.3810^{-6}$ \\
\hline
\end{tabular}

\section{IV.Discussion}

\section{Comparison with previous studies}

We find that neutral hydrogen is never stable in $\mathrm{mZrO}_{2}$ with a negative $\mathrm{U}=-2.44 \mathrm{eV}$ with hybrid functionals $(-1.36 \mathrm{eV}$ with GGA). The present work can be compared to a few studies on DFT calculations on hydrogen insertion in various phases of zirconia. Many of the older ones fail to reproduce the negative $U$ behaviour of hydrogen in zirconia, the CTL being pinned to the top of the valence band [28-31]. This probably comes from the use of less accurate local or semi-local functionals. Nevertheless, studying tetragonal $\mathrm{ZrO}_{2}$, Youssef et al[32] found a CTL in the gap with GGA, as we do. Our GGA (+/) CTL lies at $2.8 \mathrm{eV}$ a value in perfect agreement with their prediction. More recent research use hybrid functionals. They also exhibit results comparable to ours. The most direct comparison is with Lyons et al[37] who made HF calculations of the energetics of $\mathrm{H}$ in monoclinic zirconia. They find that the (+/-) CTL is at $4.1 \mathrm{eV}$ from the valence band in very good agreement with our present result of $4.0 \mathrm{eV}$. Unfortunately they did not consider neutral oxygen so they give no value for U. Marinopoulos et al [57] and Vieira et al [36] have calculated the energetics of $H$ in stabilized zirconia, with yttrium (YWZ) for the former and scandium (ScSZ) for the latter. They find values of $U$ of $-2.8 \mathrm{eV}$ in and $-3.0 \mathrm{eV}$ respectively. These values are in reasonable agreement with our value of $-2.44 \mathrm{eV}$ in view the very 
likely importance of impurities and induced oxygen vacancies in stabilized zirconia. Our results are therefore consistent with previous studies.

Concerning the insertions sites, our original systematic approach with a grid of insertion sites provides new pieces of information, especially for protons. Indeed, for hydride ions we find that there is in fact only one stable site far from any $\mathrm{Zr}$ or $\mathrm{O}$ atoms in agreement with previous works [32, 37]. However, for protons, we obtain a more complex picture. As previously guessed (all references just above), the lowest energy site is close to an oxygen atom. Yet, other metastable sites that are quite close in energy have slightly larger $\mathrm{H}-\mathrm{O}$ distances. The existence of these sites was overlooked in previous studies, which assumed that protons would sit very close to oxygen ions. Moreover, Table $2 \mathrm{~b}$ shows that the energies of these sites do not vary regularly with the $\mathrm{H}-\mathrm{O}$ distance, as one may have expected. The energetic landscape of protons in zirconia is therefore more complex than previously anticipated with 16 metastable positions of quite close energies in the unit cell of $\mathrm{m}-\mathrm{ZrO}_{2}$. The multiplicity of proton sites translates into a complex landscape for migration paths with many different migration paths.

Studies on migration energies are scarcer. To our knowledge, the only available studies on the migration energies of $\mathrm{H}$ in $\mathrm{mZrO}_{2}$ are from Muta et al[33] for neutral $\mathrm{H}$. They find a migration energy of $2 \mathrm{eV}$ comparable with our results for $\mathrm{H}^{-}$. Rabone et al[34] performed GGA-based metadynamics simulation of proton diffusion in tetragonal zirconia at $1500 \mathrm{~K}$. They obtain a diffusion coefficient of $410^{-5} \mathrm{~cm}^{2} \cdot \mathrm{s}^{-1}$ quite close to our present values at the same temperature. Indeed we obtain between $1.510^{-5}$ and $210^{-5} \mathrm{~cm}^{2} \cdot \mathrm{s}^{-1}$ in the fast diffusing directions ((001), $(010)$ and $\left.(10 \overline{3})\right)$. The proximity of the results should not be over-interpreted as both the functionals and the crystallographic structure of zirconia differ in the studies. Still the closeness is satisfactory. Finally Mantz et al [30] used GGA based molecular dynamics simulations to study the diffusion of protons. Our results are consistent with theirs. Indeed, they indicate an estimation of the migration energy of $0.32 \mathrm{eV}$ and we recalculated from their results a jump frequency of protons between neighbouring oxygen sites of $1.610^{11} \mathrm{~Hz}$ at our temperature of interest $\left(285^{\circ} \mathrm{C}\right)$. Both values fall close to our values of table $3 \mathrm{~b}$ and 4 .

A few things are worth stressing concerning the values of diffusion coefficients obtained from our calculations. First, they are non isotropic. The anisotropy is significant for hydride ions but less marked for protons. Indeed diffusion coefficient ratio amounts to around 30 between the fastest and slowest diffusion directions for protons while it exceeds 300 for hydride ions. Second, diffusion coefficients of $\mathrm{H}^{+}$are more than ten orders of magnitude faster than the ones of $\mathrm{H}^{-}$ions. This indicates that protons diffuse very fast in monoclinic zirconia unlike hydride ions that are practically trapped within the lattice. In the present case of hydrogen in pure defect-free zirconia, one can expect the Fermi level to be controlled by the presence of hydrogen in the material and the necessity for the material to be overall neutral. It should therefore be pinned at the $\mathrm{CTL}$ between $\mathrm{H}^{+}$and $\mathrm{H}^{-}$resulting in equal amounts of $\mathrm{H}^{+}$and $\mathrm{H}^{-}$. At finite temperature, the position of the $\mathrm{CTL}$ is shifted from the value indicated in table 2 by the difference in free energy of formations between $\mathrm{H}^{+}$and $\mathrm{H}^{-}$. At $285^{\circ} \mathrm{C}$ the shift is $0.62-0.45=0.18$ $\mathrm{eV}$ towards the valence band. This shift simply ensures that free energy of formations of $\mathrm{H}^{+}$and $\mathrm{H}^{-}$are equal and thereupon their respective concentrations are also equal. In short, we predict the protons to diffuse while hydride ions should remain completely immobile in comparison.

In the case of oxidized zirconium claddings, the global system (metal/oxide/water) is electrically neutral as a whole but the local neutrality is likely not true and the local concentrations of protons and hydride ions are not necessarily equal. For the diffusion coefficients of hydrogen in the zirconia layer, experimental data point to a value of the order of $10^{-14} \mathrm{~cm}^{2} \mathrm{~s}^{-1}$ at around $300^{\circ} \mathrm{C}$. This value lies in between the calculated values for $\mathrm{H}^{+}$and $\mathrm{H}^{-}$. According to the six orders of magnitude between the 
experimental value and the calculated diffusion coefficient for hydride ions, $\mathrm{H}^{-}$is certainly not responsible for hydrogen transport in the oxide layer and for the hydrogen pick-up by the metal. To reconcile our results with this value one should assume that protons are indeed the diffusing hydrogen species but that their diffusion in the oxide layer is somehow slowed down. The most likely processes for such slowing down are either the trapping of hydrogen in imperfections of zirconia or the electric field built up in the oxide layer preventing the proton migration (not discussed in this paper) $[58,59]$ The main two classes of such imperfections are point defects potentially induced by irradiation (such as oxygen vacancies ...) and alloying elements in the cladding. Indeed Zircaloy-4 and M5 alloys contain mainly tin and niobium, respectively. Hydrogen diffusion coefficient in the oxide layer formed on M5 alloy is slightly higher than the one measured on Zircaloy-4 $[13,14,16,17]$. This difference is likely due to these alloying elements.

\section{Conclusions}

From the atomic point of view, our systematic search for insertion sites and migration pathways enabled to show that the energetic landscape of hydrogen, especially protons in zirconia is more complex than anticipated. Even if simple chemical assumptions point to the most stable site, others that are very close in energy also exist. The multiplicity of stable sites for protons induces a complex kinetic landscape. The integration of all the pieces of information to obtain values of the diffusion coefficients in various directions would have been difficult without an automated tool like Kineclue.

Finally, we show that the diffusion coefficient of protons is orders of magnitude larger than that of hydride ions in monoclinic zirconia. This proves that $\mathrm{H}^{+}$is indeed the diffusing hydrogen specie in zirconia. The values of the diffusion coefficients for protons however are much larger than the values deduced from experiments. We suspect that hydrogen atoms are trapped by impurities or defects present in the oxidation layer.

\section{Appendix}

In this Appendix we provide the analytical expressions for the hydride ion diffusion coefficients in various directions as a function of jump frequencies. These expressions were obtained with the KineCluE code. In the following expressions, the diffusion coefficients are given in $\AA^{2} / s$, provided that jump probability per unit time (symbol $W$ ) are replaced by numerical values in $\mathrm{s}^{-1}$. Jump probabilities per unit time are related to jump frequencies $\Gamma$ with the relation $W_{i j}=P_{i} \Gamma_{i j}$, where $P_{i}$ is the probability of being in a state $i$ from which this particular jump from state $i$ to state $j$ can be performed, $P_{i}=$ $e^{-E_{i} / k T} / \sum_{j} e^{-E_{j} / k T}$. Here are the hydride ion diffusion coefficients in various directions:

$D_{a}\left(H^{-}\right)=\frac{88.67035 W_{13} W_{14 s}+0.76608 W_{13} W_{14 l}+52.96009 W_{14 s} W_{14 l}}{2 W_{13}+W_{14 s}+W_{14 l}}$

$D_{b}\left(H^{-}\right)=27.09642 W_{57}$

$D_{c}\left(H^{-}\right)=\frac{13.05687 W_{13} W_{14 s}+28.19484 W_{13} W_{14 l}+1.43897 W_{14 s} W_{14 l}}{2 W_{13}+W_{14 s}+W_{14 l}}$

$D_{10 \overline{3}}\left(H^{-}\right)=\frac{0.26978 W_{13} W_{14 s}+25.83097 W_{13} W_{14 l}+10.41054 W_{14 s} W_{14 l}}{2 W_{13}+W_{14 s}+W_{14 l}}$ 
Because directions $a$ and $c$ are not orthogonal to each other, there are some correlations between the displacements in these directions, and these correlated displacements can be written as a diffusion coefficient:

$D_{a c}\left(H^{-}\right)=D_{c a}\left(H^{-}\right)=\frac{34.02583 W_{13} W_{14 s}-4.64753 W_{13} W_{14 l}-8.72973 W_{14 s} W_{14 l}}{2 W_{13}+W_{14 s}+W_{14 l}}$

Let us a note an interesting limiting case occurring in this study. The above expressions become much simpler when $W_{14 s} \ll W_{13}, W_{14 l}$, and diffusion coefficients do not depend on $W_{14 s}$ anymore.

$D_{a}\left(H^{-}\right) \simeq 88.67035 W_{13}+52.96009 W_{14 l}$

$D_{b}\left(H^{-}\right) \simeq 27.09642 W_{13}$

$D_{c}\left(H^{-}\right) \simeq 13.05687 W_{13}+1.43897 W_{14 l}$

$D_{10 \overline{3}}\left(H^{-}\right) \simeq 0.26978 W_{13}+10.41054 W_{14 l}$

$D_{a c}\left(H^{-}\right)=D_{c a}\left(H^{-}\right) \simeq 34.02583 W_{13}-8.72973 W_{14 l}$

The expressions for the proton ion are much more complicated because there are many available sites that are not equivalent by symmetry, and therefore, the solution of the diffusion problem requires the inversion of a $4 \times 4$ matrix. Given the length of the expression, printing the analytical result here would not make much sense, and interested readers can directly use the KineCluE code [53] to reproduce the results.

\section{References}


1. Garner, G.L., B.A. Hilton, and E. Mader. Performance of alloy M5TM cladding and structure. in LWR Fuel Performance Meeting/Top Fuel. 2007. San Francisco.

2. Garde, A.M., Enhancement of Aqueous Corrosion of Zircaloy-4 Due to Hydride Precipitation at the Metal-Oxide Interface, C.M. Eucken and A.M. Garde, Editors. 1991, ASTM International: West Conshohocken, PA. p. 566-591.

3. Kido, T., A study on enhanced uniform corrosion of Zircaloy-4 cladding during high burnup operation in PWRS. United States: Minerals, Metals ampersand Materials Society. 1993

4. Blat, M. and D. Noel, Detrimental Role of Hydrogen on the Corrosion Rate of Zirconium Alloys, E.R. Bradley and G.P. Sabol, Editors. 1996, ASTM International: West Conshohocken, PA. p. 319-337.

5. Blat, M., et al., Contribution to a Better Understanding of the Detrimental Role of Hydrogen on the Corrosion Rate of Zircaloy-4 Cladding Materials, G.P. Sabol and G.D. Moan, Editors. 2000, ASTM International: West Conshohocken, PA. p. 563-591.

6. Kim, Y.S., K.S. Rheem, and D.K. Min, Phenomenological Study of In-Reactor Corrosion of Zircaloy-4 in Pressurized Water Reactors, A.M. Garde and E.R. Bradley, Editors. 1994, ASTM International: West Conshohocken, PA. p. 745-759.

7. Kim, Y.S., et al., The Cause for Enhanced Corrosion of Zirconium Alloys by Hydrides, G.D. Moan and P. Rudling, Editors. 2002, ASTM International: West Conshohocken, PA. p. 274-296.

8. Tupin, M., et al., Mechanism of corrosion of zirconium hydride and impact of precipitated hydrides on the Zircaloy-4 corrosion behaviour. Corrosion Science. 98 (2015) 478-493.

9. Tupin, M., et al., Hydrogen diffusion process in the oxides formed on zirconium alloys during corrosion in pressurized water reactor conditions. Corrosion Science. 116 (2017) 1-13.

10. Kunz, W., H. Münzel, and U. Kunz, Tritium release from Zircaloy-2: Dependence on temperature, surface conditions and composition of surrounding medium. Journal of Nuclear Materials. 136 (1985) 6-15.

11. Couet, A., et al., An integrated modeling and experimental approach to study hydrogen pickup mechanism in zirconium alloys. Corrosion Science. 159 (2019).

12. Aufore, L., Aix-Marseille 2 University 1997, Etude du transport de l'hydrogène produit lors de la corrosion des gaines d'éléments combustibles des réacteurs à eau sous pression dans la zircone et le Zircaloy-4

13. Khatamian, D. and F.D. Manchester, An ion beam study of hydrogen diffusion in oxides of $\mathrm{Zr}$ and $\mathrm{Zr}-\mathrm{Nb}$ (2.5 wt\%): I. Diffusion parameters for dense oxide. Journal of Nuclear Materials. 166 (1989) 300-306.

14. Khatamian, D., Hydrogen diffusion in oxides formed on surfaces of zirconium alloys. Journal of Alloys and Compounds. 253-254 (1997) 471-474.

15. Hillner, E., et al., Hydrogen absorption in zircaloy during aqueous corrosion: effect of environment. WAPD (Series) ;TM-411. Washington, D.C.: Office of Technical Services, Department of Commerce. iii, 40 pages. 1964

16. Khatamian, D., Diffusion of Hydrogen in the Oxides of $Z r-1 N b, Z r-2.5 \mathrm{Nb}$ and $\mathrm{Zr}-20 \mathrm{Nb}$ Alloys. Zeitschrift fur Physikalische Chemie. 181 (1993) 435-440.

17. Hatano, Y., et al., Transport mechanism of hydrogen through oxide film formed on zircaloy-4. Journal of Radioanalytical and Nuclear Chemistry. 239 (1999) 445-448.

18. Takagi, I., et al., Deuterium diffusion in steam-corroded oxide layer of zirconium alloys. Journal of Nuclear Materials. 419 (2011) 339-346.

19. Bisor, C., PhD thesis, Evry val d'Essonne, 2010, Compréhension des mécanismes de prise $d^{\prime}$ 'hydrogène des alliages de zirconium en situation de corrosion en conditions REP - Impact des hydrures sur la corrosion du Zircaloy-4 
20. Sundell, G., et al., Direct observation of hydrogen and deuterium in oxide grain boundaries in corroded Zirconium alloys. Corrosion Science. 90 (2015) 1-4.

21. Jublot, M., et al., Influence of Hydride Precipitation on the Corrosion Kinetics of Zircaloy-4: Effect of the Nanostructure and Grain Boundary Properties of the Zirconium Oxide Layer on Oxygen Diffusion Flux, R. Comstock and A. Motta, Editors. 2018, ASTM International: West Conshohocken, PA. p. 350-384.

22. Hu, J., et al., Hydrogen pickup during oxidation in aqueous environments: The role of nanopores and nano-pipes in zirconium oxide films. Acta Materialia. 180 (2019) 105-115.

23. Park, K. and D.R. Olander, Oxygen diffusion in single-crystal tetragonal zirconia. (1991).

24. Park, K. and D.R. Olander, Hydrogen Dissolution in and Release from Nonmetals: III, Tetragonal Zirconia. Journal of the American Ceramic Society. 74 (1991) 72-77.

25. Van De Walle, C.G. and J. Neugebauer, Hydrogen in semiconductors, in Annual Review of Materials Research. 2006. p. 179-198.

26. Kirchheim, R. and A. Pundt, Hydrogen in Metals, in Physical Metallurgy: Fifth Edition. 2014. p. 2597-2705.

27. Heyd, J., G.E. Scuseria, and M. Ernzerhof, Hybrid functionals based on a screened Coulomb potential. Journal of Chemical Physics. 118 (2003) 8207-8215.

28. Peacock, P.W. and J. Robertson, Behavior of hydrogen in high dielectric constant oxide gate insulators. Applied Physics Letters. 83 (2003) 2025-2027.

29. Xiong, K., J. Robertson, and S.J. Clark, Behavior of hydrogen in wide band gap oxides. Journal of Applied Physics. 102 (2007) 083710.

30. Mantz, Y.A. and R.S. Gemmen, Protonated Forms of Monoclinic Zirconia: A Theoretical Study. The Journal of Physical Chemistry C. 114 (2010) 8014-8025.

31. Malki, B., O. Le Bacq, and A. Pasturel, Ab initio study of hydrogen related defect in ZrO2: Consequences on dry and aqueous oxidation. Journal of Nuclear Materials. 416 (2011) 362368.

32. Youssef, M. and B. Yildiz, Hydrogen defects in tetragonal ZrO2 studied using density functional theory. Physical Chemistry Chemical Physics. 16 (2014) 1354-1365.

33. Muta, H., et al., Ab initio study of hydrogen diffusion in zirconium oxide. Journal of Nuclear Science and Technology. 49 (2011) 544-550.

34. Rabone, J. and P. Van Uffelen, DFT-based Metadynamics simulation of proton diffusion in tetragonal zirconia at 1500K. Journal of Nuclear Materials. 459 (2015) 30-36.

35. Marinopoulos, A.G., Protons in cubic yttria-stabilized zirconia: Binding sites and migration pathways. Solid State Ionics. 315 (2018) 116-125.

36. Vieira, R.B.L., et al., Isolated hydrogen configurations in zirconia as seen by muon spin spectroscopy and ab initio calculations. Physical Review B. 94 (2016) 11.

37. Lyons, J.L., A. Janotti, and C.G. Van de Walle, The role of oxygen-related defects and hydrogen impurities in HfO2 and ZrO2. Microelectronic Engineering. 88 (2011) 1452-1456.

38. Kresse, G. and J. Furthmuller, Efficient iterative schemes for $a b$ initio total-energy calculations using a plane-wave basis set. Physical Review B. 54 (1996) 11169-11186.

39. Kresse, G. and D. Joubert, From ultrasoft pseudopotentials to the projector augmented-wave method. Physical Review B. 59 (1999) 1758-1775.

40. Blochl, P.E., Projector Augmented-Wave Method. Physical Review B. 50 (1994) 17953-17979.

41. Perdew, J.P., K. Burke, and M. Ernzerhof, Generalized gradient approximation made simple. Physical Review Letters. 77 (1996) 3865.

42. Howard, C.J., R.J. Hill, and B.E. Reichert, Structures of ZrO2 polymorphs at room temperature by high-resolution neutron powder diffraction. Acta Crystallographica Section B. 44 (1988) 116120.

43. Jiang, $\mathrm{H}$., et al., Electronic band structure of zirconia and hafnia polymorphs from the \$GW\$ perspective. Physical Review B. 81 (2010) 085119.

44. Jaffe, J.E., R.A. Bachorz, and M. Gutowski, Low-temperature polymorphs of $\mathrm{ZrO}_{2}$ and $\mathrm{HfO}_{2}: \mathrm{A}$ density-functional theory study. Physical Review B. 72 (2005) 144107. 
45. Bieze, T.W.N., J.R.C. van der Maarel, and J.C. Leyte, The intramolecular $\mathrm{OH}$ bond length of water in a concentrated poly(ethyleneoxide) solution. An NMR relaxation study. Chemical Physics Letters. 216 (1993) 56-62.

46. Magnuson, M., et al., Bonding Structures of $\mathrm{ZrHx}$ Thin Films by X-ray Spectroscopy. Journal of Physical Chemistry C. 121 (2017) 25750-25758.

47. Taylor, S.E. and F. Bruneval, Understanding and correcting the spurious interactions in charged supercells. Physical Review B. 84 (2011) 075155.

48. Harrop, P.J. and J.N. Wanklyn, The dielectric constant of zirconia. British Journal of Applied Physics. 18 (1967) 739-742.

49. Ashcroft, N. and N. Mermin, Solid State Physics. 1976, Brooks/Cole.

50. Jonsson, H., G. Mills, and K.W. Jacobsen, Nudged elastic band method for finding minimum energy paths of transitions, in Classical and Quantum Dynamics in Condensed Phase Simulations. 1998, WORLD SCIENTIFIC. p. 385-404.

51. Henkelman, G., B.P. Uberuaga, and H. Jónsson, Climbing image nudged elastic band method for finding saddle points and minimum energy paths. Journal of Chemical Physics. 113 (2000) 9901-9904.

52. Vineyard, G.H., Frequency factors and isotope effects in solid state rate processes. Journal of Physics and Chemistry of Solids. 3 (1957) 121-127.

53. Schuler, T., L. Messina, and M. Nastar, KineCluE: A kinetic cluster expansion code to compute transport coefficients beyond the dilute limit. Computational Materials Science. 172 (2020) 109191.

54. Kineclue. Available from: https://github.com/lukamessina/kineclue.

55. Nastar, M., V.Y. Dobretsov, and G. Martin, Self-consistent formulation of configurational kinetics close to equilibrium: The phenomenological coefficients for diffusion in crystalline solids. Philosophical Magazine A. 80 (2000) 155-184.

56. Van de Walle, C.G. and J. Neugebauer, Universal alignment of hydrogen levels in semiconductors, insulators and solutions. Nature. 423 (2003) 626-628.

57. Marinopoulos, A.G., Incorporation and migration of hydrogen in yttria-stabilized cubic zirconia: Insights from semilocal and hybrid-functional calculations. Physical Review B. 86 (2012) 155144.

58. Couet, A., A.T. Motta, and A. Ambard, The coupled current charge compensation model for zirconium alloy fuel cladding oxidation: I. Parabolic oxidation of zirconium alloys. Corrosion Science. 100 (2015) 73-84.

59. Tupin, M., et al. Understanding of corrosion mechanisms after irradiation: Effect of ion irradiation of the oxide layers on the corrosion rate of M5. in ASTM Special Technical Publication. 2018. 